\title{
FOLDING ELASTIC TRANSMEMBRANE HELICES TO FIT IN A LOW-RESOLUTION IMAGE BY ELECTRON MICROSCOPY
}

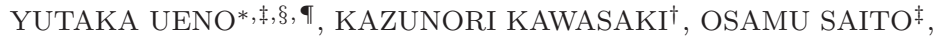 \\ MASAFUMI ARAI ${ }^{\S}$ and MAKIKO SUWA ${ }^{\S}, \|$ \\ *Biomedical Research Institute \\ National Institute of Advanced Industrial Science and Technology (AIST) Tsukuba \\ 1-1 Umezono Central-2, Tsukuba, Ibaraki, 305-8568 Japan \\ ${ }^{\dagger}$ Health Research Institute, AIST Kansai \\ 1-8-31 Mirodigaoka, Ikeda, Osaka 563-8577 Japan \\ ${ }^{\ddagger}$ Graduate School of Information Science \\ Nara Institute of Science and Technology, 8916-5 Takayama-cho \\ Ikoma-shi, Nara 630-0192, Japan \\ $\S_{\text {Computational Biology Research Center, AIST Tokyo Waterfront }}$ \\ 2-4-7 Aomi, Koto-ku, Tokyo, 135-0064 Japan \\ 『uenoyt@ni.aist.go.jp

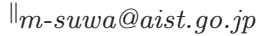

Received 25 July 2011

Revised 2 September 2011

Accepted 15 September 2011

\begin{abstract}
Structure prediction of membrane proteins could be constrained and thereby improved by introducing data of the observed molecular shape. We studied a coarse-grained molecular model that relied on residue-based dummy atoms to fold the transmembrane helices of a protein in the observed molecular shape. Based on the inter-residue potential, the $\alpha$-helices were folded to contact each other in a simulated annealing protocol to search optimized conformation. Fitting the model into a three-dimensional volume was tested for proteins with known structures and resulted in a fairly reasonable arrangement of helices. In addition, the constraint to the packing transmembrane helix with the twodimensional region was tested and found to work as a very similar folding guide. The obtained models nicely represented $\alpha$-helices with the desired slight bend. Our structure prediction method for membrane proteins well demonstrated reasonable folding results using a low-resolution structural constraint introduced from recent cell-surface imaging techniques.
\end{abstract}

Keywords: Protein folding; protein structure prediction; transmembrane helix; coarsegrained molecular dynamics; off-lattice model.

${ }^{\top}$ Corresponding author. Current address: Health Research Institute, AIST Amagasaki, 3-11-46 Nakoji, Amagasaki, Hyogo 661-0974, Japan. 


\section{Introduction}

For understanding functions of membrane proteins, both structure determination and the $a b$ initio structure prediction of them from amino acid sequences are formidable tasks. In practical drug design studies, large numbers of projects have applied homology modeling and molecular dynamics simulations to meet the demand of structure prediction of membrane proteins. ${ }^{1}$ To fold proteins with the molecular dynamics, the coarse-grained molecular model has been a practical choice to reduce computational cost.

In our study of protein folding simulation, we incorporated experimental data regarding the molecular shape of membrane proteins observed on membrane by electron microscopy. The current study is targeted at proteins with transmembrane helices; in particular, G-protein-coupled receptors (GPCRs), have been well studied for structure prediction from primary sequences. Recently, not only novel crystallographic models but also low-resolution structure information on the membrane proteins have increased. Historically, the image of cell surfaces by electron microscopy has provided fundamental understanding of membrane proteins and their molecular interactions. ${ }^{2}$ Because of the fragile nature of biological macromolecules, the resolution stays at several nanometers. Instead of annotating these proteins on a purely speculative basis, experimental labeling techniques using immunoglobulin can determine the localization and distribution of a target protein, albeit at substantial experiment effort. Figure 1 is an example of cell-surface observation by the freeze-fracture replica image of the yeast, Candida tropicalis. ${ }^{3}$ The image quality was dramatically improved by experimental techniques. ${ }^{4}$ The gray-scale image represents the thickness of the replica, and the detailed molecular conformation is modulated by coating platinum and other phenomena. Even though the preserved shape of the protein revealed the expressed membrane proteins in the cell,

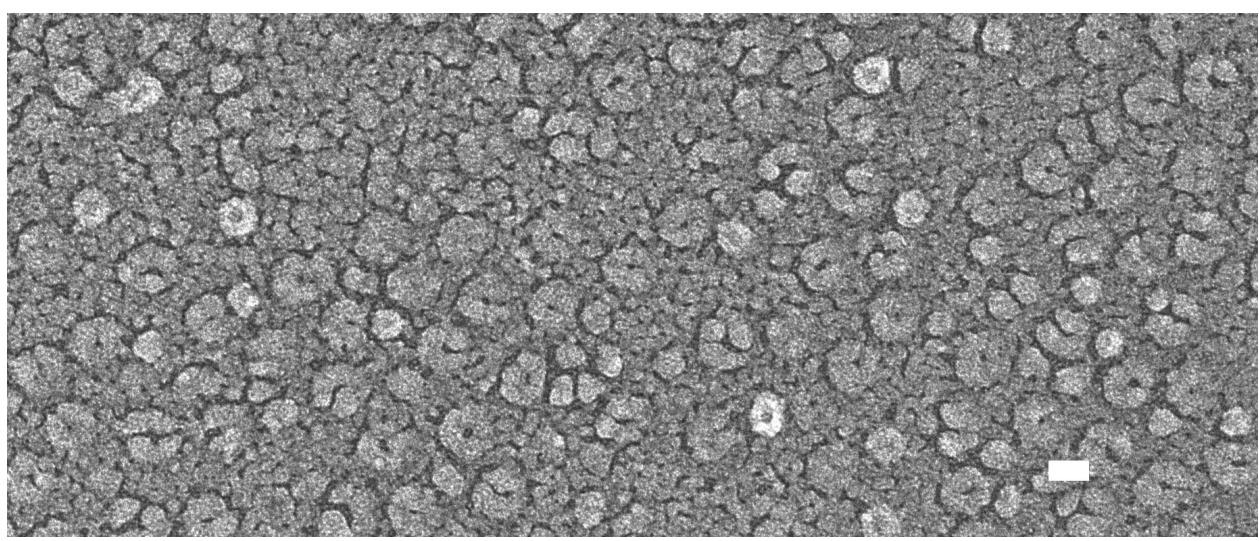

Fig. 1. A typical image of yeast cell surface by freeze-fracture replica electron microscopy. The thickness of the platinum coat is $\sim 4 \mathrm{~nm}$, inflating the observed shape of membrane proteins. Scale bar, $10 \mathrm{~nm}$. 
membrane proteins prevented us from performing a proteomics study by the mass spectroscopy utilized widely for cases with soluble proteins.

For the challenge of annotating membrane proteins on membrane surface using cell observations by electron microscopy, we studied a three-dimensional modeling tool to fit the observed molecular shape data. So far, the two-dimensional analysis of the packing of transmembrane helices at a cross-section of the membrane ${ }^{5}$ has provided key data on the interaction of amino acid residues for structure prediction. In many previous structure-prediction studies, transmembrane helices have been modeled with solid cylinders. ${ }^{6,7}$ On evaluating contacting amino acid residues on the transmembrane helices, using a solid cylinder template that differs from a real protein conformation is a cause of mismatch. In addition, a full parameter search for the optimum helix bundling geometry is a computational burden. ${ }^{6}$ This paper introduces a modeling tool for transmembrane helices allowing slight bending using coarse-grained molecular dynamics. With transmembrane helices predicted from amino acid sequences, a folding simulation was demonstrated to find an optimized fold.

Figure 2 illustrates different scenarios of folding simulation: a theoretical structure prediction only with property of the amino acid residues is time consuming (Fig. 2(a)), a low-resolution electron density map of transmembrane helices has allowed building an atomic model by a molecular dynamics simulations (Fig. 2(c)). Our structure prediction method resides in between these two approaches: folding with a constraint for the transmembrane helices (Fig. 2(b)). This paper demonstrates our coarse-grained molecular dynamics to finds possible arrangement of transmembrane helices using volume constraint in these three scenarios. In particular for GPCR, knowledge of informatics analysis obtained from comparative

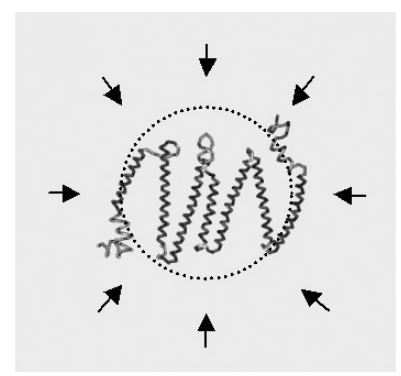

(a)

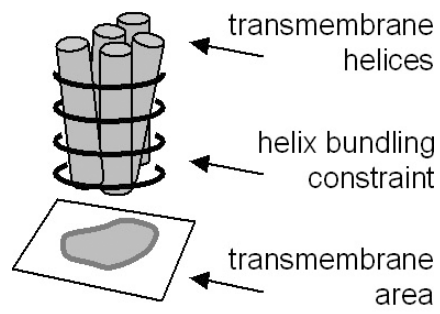

(b)

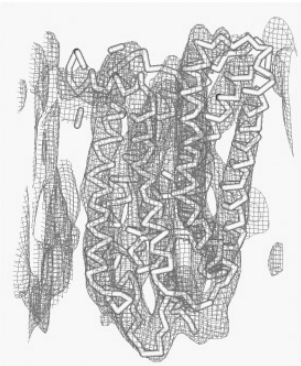

(c)

Fig. 2. Packing transmembrane helices by folding simulation. There are some approaches to fold peptide chains with predicted transmembrane helices. (a) A free-folding simulation only with a globular shape constraint. Arrows indicate interactions from solvent molecules that drive folding into a globlar shape. (b) A constraint of membrane region is applied to the transmembrane helices. (c) A volumetric density map provides constraint to build an atomic model by existing modeling method. A map for bovine rhodospin by electron crystallography from EM database EMD1079 and corresponding atomic model. 
genomics analysis among various species has already provided a rich resource for structure prediction. ${ }^{9}$ We consider that a whole catalogue of putative GPCR models is a practical step toward constructing an atlas of membrane proteins in the cells based on annotations of observed cell surface images.

\section{Methods}

\subsection{Molecular shape data}

First, the following summaries will clarify the two types of structural information: three-dimensional volume data and the two-dimensional area of transmembrane helices.

\subsubsection{Low-resolution volume data of membrane proteins by electron microscopy}

Single-particle analysis of proteins using transmission electron microscopy has been employed for membrane proteins solubilized by a detergent. Using a dilute solution of protein, the three-dimensional volume of the molecule is reconstructed from thousands of images representing different projections of the molecule. Staining with a heavy-metal solution is useful in increasing the contrast of images, but recent techniques of cryo-electronmicroscopy allow imaging of intact specimens by quick freezing. The preparation of membrane protein specimens requires substantial biochemical studies that stand in the way of high-throughput structure analysis. The EM databank (http://emdatabank.org) provides a repository of the reconstructed volumetric data available for a structure prediction study.

\subsubsection{Membrane imaging by freeze-fracture replica electron microscopy}

Using a specimen of the membrane from in vivo cells, a drop of the solution containing the specimen was quickly frozen by a metal block cooled by liquid helium. Then the specimen was fractured to expose both hydrophobic faces of the lipid bilayer membrane. A replica of the fractured half membrane was made taken by a thin coating with evaporated platinum, and backed with evaporated carbon. Then the surface of replica was observed by transmission electron microscopy. The special resolution of obtained cell images is at most $4 \mathrm{~nm}$ depending on various experimental factors. ${ }^{2}$ In addition, atomic force microscopy also provides cell surface images from the aqueous environment.

\subsection{A coarse-grained polypeptide model and the folding potential}

Among variations of coarse-grained molecular model and force field potential for proteins, ${ }^{10}$ we used a simple off-lattice one-bead model similar to the reference. ${ }^{11}$ The dummy atoms representing amino acid residues are connected without the 
stereochemistry constraint of the peptide chain, which is expected to be counterbalanced by the non-bonded potential. We consider this coarse graining to be tolerable if our target resolution of the model is around $0.6 \mathrm{~nm}$, where the coordinates of two interacting amino acid residues are blurred. Motions of the dummy atom are calculated by classical Newton equations with the force derived from the potential energy that is sum of interaction potentials:

$$
\begin{aligned}
& E_{\text {total }}=E_{\text {bond }}+E_{\text {nonbond }}+E_{\text {constraint }} \\
& E_{\text {bond }}=\sum_{i, j}^{\text {bond-pairs }} k\left(r_{i j}-a\right)^{2}
\end{aligned}
$$

The bond length $a$ between dummy atoms is usually $\sim 0.36 \mathrm{~nm}$. Currently, the bond constant $k$ is unity, which could be scaled to conventional stereochemical energy.

\subsubsection{The inter-residue potential}

The pairwise inter-residue potential representing repulsion and attraction ${ }^{12}$ is employed using the same constant $c^{a b}$ for each amino acid $a, b$ of the dummy atoms pairs $i, j$. The distance-dependent function of this Sippl-like potential is approximated by Lennard-Jones potential function in order to facilitate calculation of the derivative.

$$
E_{\text {nonbond }}=\sum_{i \neq j} \begin{cases}c^{a b} \cdot\left[\left(\frac{e}{r}\right)^{12}-2\left(\frac{e}{r}\right)^{6}\right] & \left(c^{a b}>0\right) \\ \left|c^{a b}\right| \cdot\left[\left(\frac{e}{r}\right)^{12}-2\left(\frac{e}{r}\right)^{6}+\frac{b}{(r-h)^{3} / d+1}\right] & \left(c^{a b}<0\right)\end{cases}
$$

Through folding simulations for various proteins with careful inspections of the motion of the model, empirical values were obtained: $b=\sim 1.7, d=\sim 15, e=$ $\sim 7, h=\sim 6.6$ as illustrated in Fig. 3, where the optimum distance between residues is $e$. The $c^{a b}$ values were scaled to 0.7 to balance with $E_{\text {bond }}$.

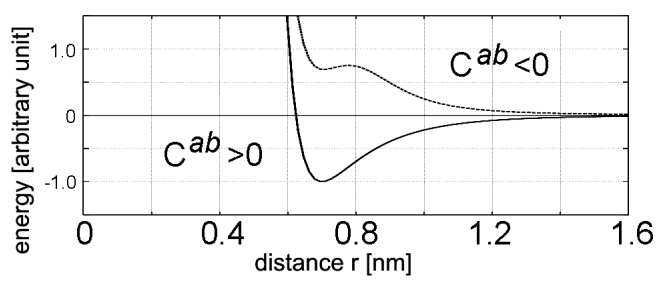

Fig. 3. The inter-residue potential. Proposed potential function fairly represents the inter residue potential by Casari and Sippl (1992). Their linear approximation fits to this function in the region from 0.7 to $1.0 \mathrm{~nm}$. 


\subsubsection{The helix-retaining potential}

The residues predicted as the $\alpha$-helix regions preserve the helix shape during the folding simulation. Unlike previous off-lattice models ${ }^{11}$ that takes preferred bond angles, following pseudo-bonds as in Eq. (2) were introduced in $E_{\text {constraint }}$ to represent a helix allowing smooth bending:

- Four pseudo-bonds toward residues $(i+2),(i+3),(i+4)$, and $(i+7)$, for the $i$ th dummy atom.

- A pseudo-bond between start and end residues of the helix.

Without the second constraint, once a helix break occurs in a simulation, the helix is rarely recovered. The coordinates are generated from the standard $\alpha$-helix model. The non-bonded interaction potential was exempted for those pairs. The binding energy constant $k$ controls the stiffness of the helix, and the value same as the peptide bond showed reasonable elastic property.

\subsubsection{The shape constraint potential}

To fold the peptide chain model into a three-dimensional volumetric data, a simple constraint potential in the same form as Eq. (2) was added to $E_{\text {constraint. The }}$ constraint area was represented as arrays of anchors within the volume at a grid interval $\sim 0.6 \mathrm{~nm}$. A bonding pair was generated for all dummy atoms and the closest anchor. The threshold distance was set to $\sqrt{3} / 2$ of the grid interval. For all bonding pairs with distances larger than the threshold, a pseudo-bond was added with its energy constant $k=\sim 0.03$, which should be tuned in the folding simulation. If the three-dimensional molecular shape is accurate enough, the model may also be fitted evenly among the anchors. Such a constraint is also possible by adding additional bonding pairs for all anchors between the closest dummy atoms.

\subsection{Simulated annealing for a folding simulation}

The model follows an ordinary molecular dynamics calculation using the velocity Verlet method for integrating the differential equation. ${ }^{13}$ The mass of the dummy atom was one-hundredth of the molecular weight of the amino acid, and the timestep of the integral was 0.2 , which is sufficient for some harmonic motions of this dummy atom system. Using a dumping factor $\sim 0.980$, the temperature of the system easily cools down within hundreds of time counts. The cut-off distance for non-bonded dummy atoms was $2.0 \mathrm{~nm}$. A simple annealing schedule was repeated several times by a heating duration of 100 time-steps at every 1000 steps (with random modification to the velocity of the dummy atoms using the dumping factor 1.0). The shaking effect of the molecule beyond a local minimum was inspected by a movie as well as the calculated temperature and total energy. 
The simulation code was implemented by programming Language Lua, with molecular graphics program MOSBY. ${ }^{14}$ Computational times required for folding 348 residues was $\sim 100$ minutes on a single microprocessor.

\section{Results}

\subsection{Packing transmembrane helix on the cross section of the membrane}

Our early attempt to pack transmembrane helices in a two-dimensional cross-section of the membrane encountered some difficulties. In Fig. 4, the transmembrane helix was represented as a circle of radius $0.5 \mathrm{~nm}$, which was viewed from the axis perpendicular to the membrane plane. A theoretical boundary shape of the membrane region was synthesized from the known atomic coordinates of bovine rhodopsin. With the optimum inter-surface distance to the adjacent helices of $\sim 1.0 \mathrm{~nm}$ on the cross-section of the membrane, all helices were moved to attract each other to make a bundle with interaction using the Lennard-Jones potential. Two optimum packing results are illustrated: Fig. 4(a) is the energy minimum compact layout; Fig. 4(b) is a layout matched to the true conformation. We considered that amino acid interactions between adjacent transmembrane helices are necessary to obtain correct layout model at the energy minimum. Furthermore, these interactions differ in the series of cross-sections, where the helix positions are altered if a helix is tilted and bent. Thus, we introduced the three-dimensional model of transmembrane helices to pack taking into account of contacting amino acid residues.

\subsection{An observation of the transmembrane helix}

To develop our three-dimensional modeling method, we observed packing of transmembrane helices in PDBTM database8. From 199 PDB entries of non-redundant sequences with 1411 transmembrane helices, their geometrical properties are summarized.

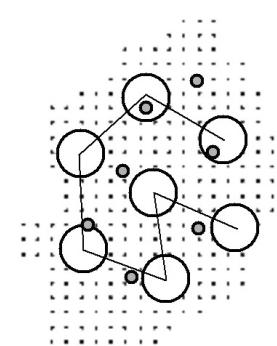

(a)

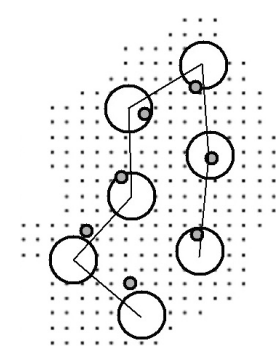

(b)

Fig. 4. Packing a cylinder model of the transmembrane helices. An example of a search in twodimensional cross-section of the membranes. Large circles indicate the estimated transmembrane helicies, and small circles indicate the correct positions. (a) The energy minimum model. (b) A model the most close to the correct packing. 
(1) Distance between transmembrane helices: Among total 7024 pairs, 961 pairs were marked as closest for each helix, and their inter-helix axis distances were mostly about $1.0 \mathrm{~nm}$, up to $1.6 \mathrm{~nm}$. Other pairs also populated around $\sim 19.0 \mathrm{~nm}$, and $78 \%$ of them reside up to $3.5 \mathrm{~nm}$.

(2) Tilt angle of transmembrane helices: The tilt angle of the transmembrane helices from the membrane normal axis was distributed around the most populated peak at $\sim 25^{\circ}$ between $15^{\circ}$ and $40^{\circ}$ by $67 \%$ of them. When the angle between two helices of all combination was measured, the distribution was much broader around the median at $\sim 30^{\circ}$.

(3) Loop region between transmembrane helices: There is no correlation between the length of the loop region and the axial distance between helices of each side. About $64 \%$ of loops between helices were made of less than 30 residues, and longer loops have little influence on the inter-axis distance of the helices.

Even though these limited the parameter ranges for the arrangement of the helix, the direct search of parameters in six degrees of freedom for all helices made the search procedure extraordinarily time consuming. Thus, we employed a folding simulation to shortcut the search procedure through a vast range of parameter space. Our coarse-grained model is designed to satisfy the above-mentioned properties of predefined $\alpha$-helices that exhibit residues which interact with other residues on adjacent helix. The loop regions will be modeled differently after the locations of transmembrane helices were optimized.

\subsection{Folding a polypeptide chain into a globular shape}

The model and simulated annealing code were tested to work as a simple freefolding simulation of proteins. From the primary sequence of bovine rhodopsin, the transmembrane helix regions were predicted by the TmHMM. ${ }^{15}$ Transmembrane helices are roughly gathered with neighboring helices being anti-parallel, and the loop region between helices was modeled as a random walk. Since this folding simulation searches a local energy minimum overtaking some energy barriers by annealing, the obtained low-energy conformation depends on the initial model. Starting from candidates with various packing of the transmembrane helices, altering the helix faces, the obtained local minimum models were sorted to obtain the best model.

This is a case without any prior shape information to the molecule. Because the non-bonded interaction for the dummy atom is very small for largely distant pairs, folding proteins into a globular shape may require the effect of solvent molecules at room temperature and atmospheric pressure. Such simulation environment was made with positional constraints to force a protein to a globular shape as in Fig. 2(a). The constraint energy to the spherical shape was introduced by the anchor grid at an interval of $0.9 \mathrm{~nm}$ as described in the Methods section. By visual inspection, folding with smooth acceleration by this term was confirmed. Once an atom gets inside the sphere, the volume constraint is removed and the non-bonded 
interaction can work to find the optimum conformation of the molecule. Although compact fold is obtained, the transmembrane helix fold was frequently inadequate.

\subsection{Folding with a three dimensional density map}

In another scenario of folding a peptide with a three-dimensional map, it was not trivial for this method to locate helix positions correctly. Using a reported density map at resolution $\sim 0.6 \mathrm{~nm}$ on EMDatabank (http://emdatabank.org), density anchor pins were generated on the grid. Figure 5 is a result of rhodopsin showing some mismatches in finding the correct conformation. The tilt angle and bend of the helices seemed reasonable compared to the native rhodopsin structure.

- The fitness depends on the initial model and the arrangement of the transmembrane helix, i.e. rotation, the polarity of the helix, and the layout of the helices.

- For determining the layout of transmembrane helices, a map with individual helix densities presents detailed information so that the conformational search sometimes fall into a local optimum.

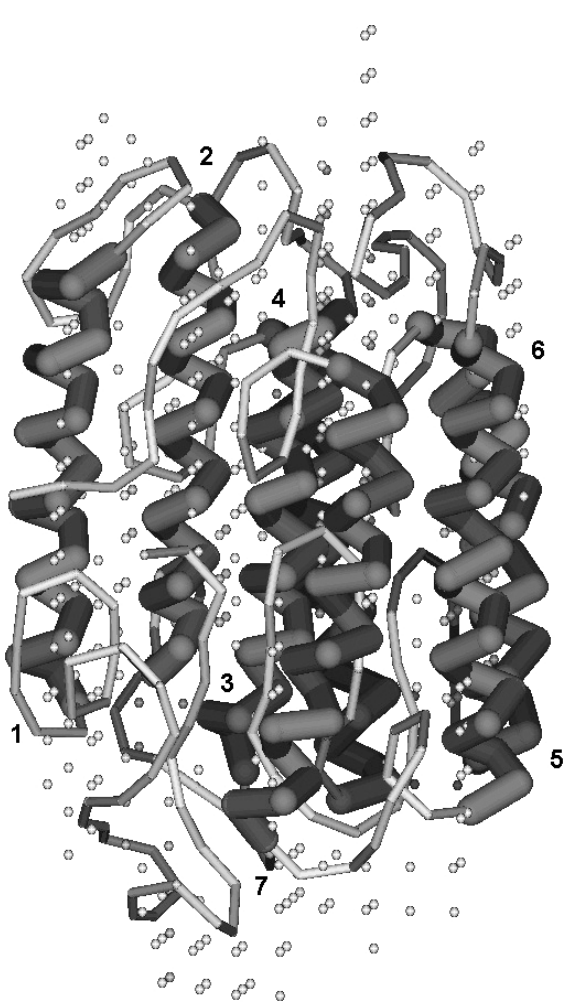

Fig. 5. A folding example of rhodopsin with volume constraint. An optimum model in this folding simulation. The small points represent the density anchor pins on a 0.3-nm grid forming the three-dimensional volumetric area for bovine rhodopsin from EMdatabase EMD1079. 
The early stage of folding needs to follow the global shape of the transmembrane region, ignoring the peak density of each helix or other domains. Then, the fitness to the high-resolution map should be examined. Otherwise, more heating should be applied, but this would run the risk of catastrophic unfolding.

\subsection{Folding with a transmembrane shape constraint}

Our current structure prediction method uses the constraint on a region of transmembrane helices (Fig. 2(b)). It is based on the proposition that once helices are bundled together, the interactions between them will drive a correct packing layout without detailed shape information. The density anchors are generated in a cylinder of radius $3.6 \mathrm{~nm}$ and height $5.2 \mathrm{~nm}$. A dummy atom of the transmembrane helix at distances of more than $0.7 \mathrm{~nm}$ from the closest anchor pin is constrained. They are exempted from the constraint if they reach the interior of the cylinder. For the loop regions, another constraint to keep them apart from the transmembrane region was applied. If they come within $0.4 \mathrm{~nm}$ of an anchor pin in membrane area, a repulsive bond between the atom and the anchor with a bond length of $1.2 \mathrm{~nm}$ is applied.

A folding result in Fig. 6(a) showed very similar shape to the reported crystallographic model, but a mismatch with the true conformation remained in the

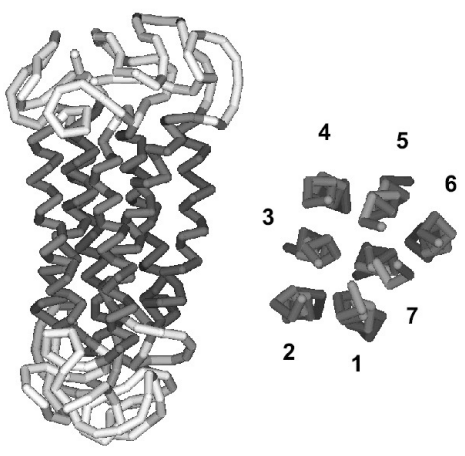

(a)

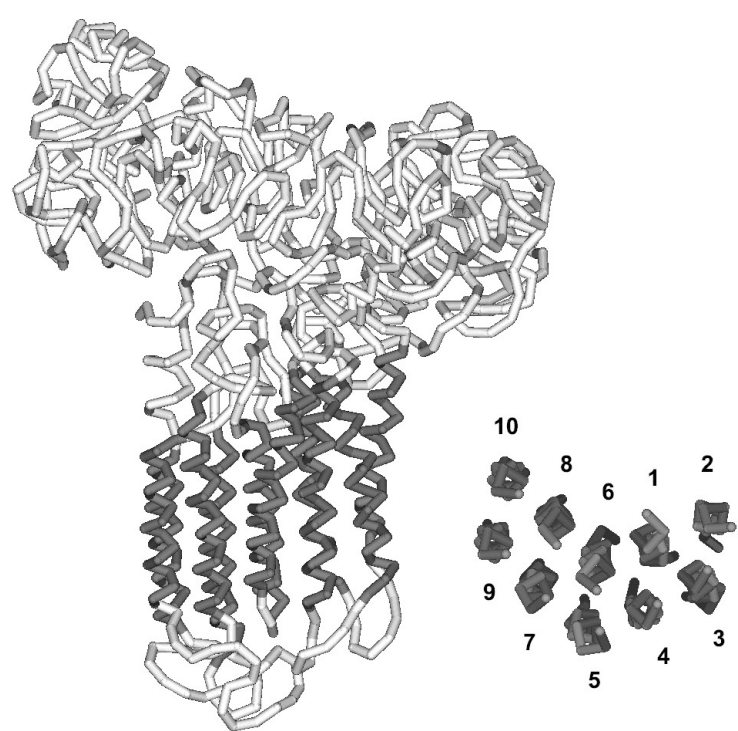

(b)

Fig. 6. Folding examples with constraints of the cylindrical transmembrane area. (a) A bovine rhodopsin model. The helix 5 should be outer. (b) Na-K-ATPase. In this fold, the helices 1 and 2 are swapped, and the helix 10 should be on the left. The regions predicted as $\alpha$-helix in the loop between transmembrane helices were also maintained. Although the obtained packing is compact, discrepancies should also be mended by detailed comparison in subsequent modeling for both model. 
location of helix 5 for bovine rhodopsin. The calculated conformational energy reached to $\sim-112.0$, which was even lower than that form a PDB conformation, whose energy was minimized into -65.0 in this potential. An unfavorable conformation as in Fig. 4(a) gave conformational energy -96 , which was higher than the optimum. To overcome some competitive local minimums, the potential still need improvements to provide its minimum at the correct conformation.

Another example of Na-K-ATPase, with predicted 10 transmembrane helices resulted in a fair fold (Fig. 6(b)), but more mismatches. These models still require subsequent modeling but provided reasonable starting conformation, including the loop regions between transmembrane helices. Folding movies are available at our web site (http://www.cbrc.jp/ ueno/mosby/copley.html).

\section{Discussion}

\subsection{Future studies}

Our work suggested that the inter-residue potential for the coarse-grained molecular model made for general proteins worked well for membrane proteins. The refinement in the potential specific to the membrane proteins or a scoring function ${ }^{16}$ should be tested with comparisons to the all-atom calculation of the stereo-chemical conformational energy using proteins whose atomic structure have been solved. The knowledge of the essential triplet amino acid interactions between transmembrane helices ${ }^{17}$ would be examined on this model with flexible helices. To speed up the conformational search, the number of initial models should be reduced. A $\beta$-sheet model would also be possible by introducing another distance constraint based on the theoretical prediction from the primary sequence. Improvements in the folding simulation for GPCR and subsequent refinement methods are currently being developed together with SEVENS database (http://sevens. cbrc.jp). ${ }^{9}$

\subsection{Atomic models and low-resolution molecular images}

The resolution of electron microscopy images of proteins is usually behind atomic resolution due to the lowdose condition, which minimizes damage to proteins. However, pioneering studies have demonstrated transmembrane helices as density rods that allowed searching helix layout by Monte Carlo assembly approach. ${ }^{18}$ Rosetta, a coarse-grained molecular dynamics approach, was also shown to fit a molecular model into the volume data. ${ }^{19}$ Unfortunately, obtaining a density map at several nanometers resolution suitable to these works is still a burden for the biologist. Rather than extracting high-resolution structure with atomic coordinates from the density map guided by modeling, our method attempted structure prediction assisted by experimental information as a constraint. 


\subsection{Visual proteomics of membrane protein}

Recent advances in tomographic reconstruction with transmission electron microscopy successfully visualized molecular details from in vivo specimens at several nanometers resolution. ${ }^{20}$ They proposed annotating proteins in the visualized volume of the specimen by assigning the shape of known proteins. With such a strategy, our structure prediction could supply putative volumetric data for proteins expected in the observation.

The oligomer formation of membrane proteins makes our method problematic because the monomer shape cannot be obtained. In that case, symmetric units could also be incorporated to our model to fit oligometric shape information.

Optimizing multiple proteins simultaneously in an observed molecular complex would be an intriguing technique. For ryanodine receptor, a Ca pump on the membrane forming a molecular complex with other proteins in various organisms. With the constituent protein structures available, a theoretical atomic model was proposed from inter-protein interaction data gathered in molecular biology studies. ${ }^{21}$ Together with the proteomics approach, these molecular simulations will meet the challenging problems of molecular biology regarding large-scale super macromolecular complexes.

\section{Conclusion}

A coarse-grained molecular model was demonstrated to simulate folding of the membrane protein guided by a low-resolution shape constraint. The method demonstrated reasonably predicted structures of membrane proteins making use of lowresolution molecular shape observations on the cell surface.

\section{References}

1. Elofsson A, von Heijne G, Membrane protein structure: Prediction versus reality, Annu Rev Biochem 76:125-140, 2007.

2. Heuser J, Preparing biological samples for stereo-microscopy by the quick-freeze, deep-etch, rotary-replication technique, Methods Cell Biol 22:97-122, 1981.

3. Osumi M, Ultrastructure of yeast: Cell wall structure and formation, Micron 29:207233, 1998.

4. Kanasaki T, Kawasaki K, Murata M, Ikeuchi Y, Ohnishi S, Structural features of membrane fusion between influenza virus and liposome as revealed by quick-freezing electron microscopy, J Cell Biol 137(5):1041-1056, 1997.

5. Suwa M, Hirokawa T, Mitaku S, A continuum theory for the prediction of lateral rotational position of $\alpha$-helices in membrane proteins, bacteriorhodopsin, Proteins 22:363-377, 1995.

6. Bowie JU, Helix-bundle membrane protein fold templates, Protein Sci 8:2711-2719, 1999.

7. Sale K, Faulon JL, Gray GA, Schoeniger JS, Young MM, Optimal bundling of transmembrane helices using sparse distance constraints, Protein Sci 13:2613-2627, 2004 . 
8. Tusnady GE, Dosztani Z, Simon I, Transmembrane proteins in the Protein Data Bank: Identification and classification, Bioinformatics 20(17):2964-2972, 2004.

9. Suwa M, Sugihara M, Ono Y, Functional and structural overview of G-proteincoupled receptors comprehensively obtained from genome sequences, Pharmaceuticals 4(4):652-664, 2011.

10. Valentina T, Coarse-grained models for proteins, Curr Opin in Struc Biol 15:144-150, 2005.

11. Guo Z, Thirumalai, Kinetics and thermodynamics of folding of a de Nove designed four-helix bundle protein, J Mol Biol 263:323-343, 1996.

12. Casari G, Sippl MJ, Structure-derived hydrophobic potential-hydrophobic potential derived from X-ray structure of globular proteins is able to identify native folds, J Mol Biol 224:725-732, 1992.

13. Komeiji Y, Ewald summation and multiple time step methods for molecular dynamics simulation of biological molecules, J Mol Struct Theo Chem 530:237-243, 2000.

14. Ueno Y, Asai K, A new plug-in software architecture applied for a portable molecular structure browser, Proc Int Conf Intell Syst Mol Biol, pp. 329-332, 1999.

15. Krogh A, Larsson B, von Heijne G, Sonnhammer ELL, Predicting transmembrane protein topology with a hidden Markov model: Application to complete genomes, J Mol Biol 305:567-580, 2001.

16. Fleishman SJ, Harrington S, Friesner AR, Honig B, Ben-Tal N, An automatic method for predicting transmembrane protein structures using cryo-EM and evolutionary data, Biophys J 87:2448-3459, 2004.

17. McAllister SR, FloudaMac CA, $\alpha$-Helical topology prediction and generation of distance restraints in membrane proteins, Biophys J 95(11):5281-5295, 2008.

18. Lindert S, Staritzbichler R, Wotzel N, Karakas M, Stewart PL, Meiler J, EMFold: De novo folding of $\alpha$-helical proteins guided by intermediate-resolution electron microscopy density maps, Structure 17(7):990-1003, 2009.

19. DiMaio F, Tyka MD, Baker ML, Chiu W, Baker D, Refinement of protein structures into low-resolution density maps using rosetta, J Mol Biol 392(1):181-190, 2009.

20. Nickell S, Kofler C, Leis AP, Baumeister W, A visual approach to proteomics, Nat Rev Mol Cell Biol 7(3):225-230, 2006.

21. Song DW, Lee JG, Youn HS, Eom SH, Kim DH, Ryanodine receptor assembly: A novel systems biology approach to 3D mapping, Prog Biophys Mol Biol 105(3):145$161,2011$.

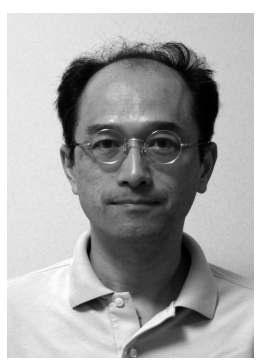

Yutaka Ueno received his Ph.D. degree in Science from Biophysical Engineering, Osaka University, Japan, in 1996. His professional career began in 1987, when he worked on molecular simulations at a private company, and bioinfomatics research at Electrotechnical Laboratory, a government research institute now renamed as National Institute of Advanced Industrial Science and Technology (AIST). From 2001 to 2008, he worked at the Computational Biology Research Center (CBRC) in AIST to develop methods of protein structure analysis using electron microscopy. Currently, he is in the Biomedical Research Institute in AIST. 

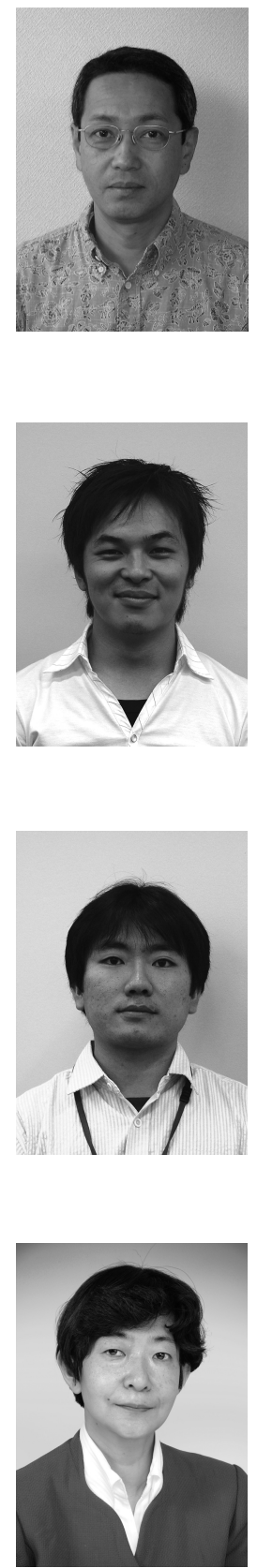

Kazunori Kawasaki received his Ph.D. degree in Biophysics from Faculty of Science, Kyoto University, Japan, in 1985. From April 1985 to March 1989, he worked as a post-doctoral fellow in Faculty of Science, and Institute of Virus Research, Kyoto University. From April 1989, he started bioimaging by fluorescence and electron microscopies at National Chemical Laboratory, the government research institute now renamed as AIST, and currently is in the Health Research Institute in AIST.

Osamu Saito received is M.E. degree in Computer Science from Graduate School of Information Science, Nara Institute of Science and Technology, Japan, in 2007. He joined the research project at CBRC in AIST to complete his thesis. Currently, he is a researcher in a private company for computational analysis and signal processing in Medical ICT.

Masafumi Arai received his Ph.D. degree in Life Science from Graduate School of Life Sciences, Tohoku University, Japan, in 2006. He was a post-doctoral fellow at CBRC in AIST from 2006 to 2007, and initiated this structure prediction study of membrane proteins. Currently, he works for a private company as an engineer for computational statistic and data analysis in pharmaceutical development.

Makiko Suwa received her Ph.D. degree in Science from Graduate School of Science and Engineering, Aoyama Gakuin University, Japan, in 1995. After her professional activities at Faculty of Engineering, Tokyo University of Agriculture and Engineering; Helix Research Institute Inc.; and Electrotechnical Laboratory, AIST, she has been leading a research team of informatics on membrane protein at CBRC, AIST, and was also a deputy director of CBRC in 2003. Currently she is a Principal Research Scientist of CBRC, AIST. 